\title{
Perioperative and Early Renal Functional Outcomes of Laparoscopic and Open Partial Nephrectomy in Clinical T1 Renal Carcinoma
}

\author{
(D) Erkan Merder, (D) Ahmet Arıman \\ University of Health Sciences Turkey, Prof. Dr. Cemil Taşşıoğlu City Hospital, Clinic of Urology, Istanbul, Turkey
}

\section{Abstract}

Objective: To compare the perioperative and postoperative outcomes of open partial nephrectomy (OPN) and laparoscopic partial nephrectomy (LPN) in clinical T1 renal carcinoma.

Methods: We examined the records of patients who underwent partial nephrectomy at our clinic between January 2016 and May 2020. The records of 20 patients who underwent LPN were compared with the records of 50 patients who underwent OPN. The demographic findings, tumor size, operation time, warm ischemia time (WIT), PADUA nephrometry score, complications, blood loss, preoperative and early postoperative estimated glomerular filtration rate (eGFR), creatinine, and hemoglobin ( $\mathrm{Hb}$ ) values, surgical margins, and hospital stay time of both surgical techniques were compared. Student's t-test and Mann-Whitney U test were used in the statistical analysis. A $p$ value $\leq 0.05$ was considered significant.

Results: Of the 70 patients, 45 were men and 25 were women, with 42 patients diagnosed with right-sided renal cell carcinoma (RCC) and 28 patients diagnosed with left-sided RCC. The median age was 55 years. Fifty-four patients were diagnosed with clinical T1a RCC, and 16 patients were diagnosed with clinical T1b RCC. The median WIT was shorter in the LPN group. The median tumor size was larger in the OPN group. There was a statistically significant difference between PADUA score and operation time in OPN, while there was no significant difference between OPN and LPN in terms of preoperative and early postoperative eGFR, creatinine, and Hb values. In OPN group, the postoperative creatinine increase was statistically significant compared with preoperative value. This difference was not statistically significant in the LPN group. Clavien-Dindo complications and hospital stay time were higher in OPN.

Conclusion: LPN is an alternative technique to OPN for treatment of clinical T1 RCC when performed by experienced urologists in experienced clinics. It was concluded that postoperative early renal function is better in LPN.

Keywords: Partial nephrectomy, RCC, laparoscopic surgery, acute kidney injury

\section{INTRODUCTION}

Renal cell carcinoma (RCC) constitutes $2 \%-3 \%$ of all cancers (1). Radiologic imaging techniques have recently advanced, and due to this development, we can diagnose kidney tumors in early stages compared with previous times $(2,3)$. Recently, partial nephrectomy ( $\mathrm{PN}$ ) is considered the most prominent method for treating CT1 kidney tumors (3).
The main purpose of PN in RCC treatment is to protect the kidney parenchyma as much as possible and reduce the decrease in glomerular filtration. In approximately $20 \%$ of patients, acute kidney injury (AKI) develops after PN (4).

Laparoscopic PN (LPN) has become the standard surgical treatment for T1a and some T1 b tumors in recent years (5). There are many studies comparing the oncologic and functional results 
of LPN and open PN (OPN) for renal masses in the literature. It was found that the results of both techniques are comparable, and LPN has some advantages over OPN (6-9).

According to the recent literature, there was no definite superiority between OPN, LPN, and robotic PN methods in terms of perioperative outcomes and AKI (10).

We analyzed the perioperative and early renal functional outcomes regarding AKI in our patients who performed LPN and OPN for treatment of clinical T1 RCC.

\section{METHODS}

This is a retrospective single-center study (Prof. Dr. Cemil Taşcıoğlu City Hospital, İstanbul, Turkey), including 86 patients who underwent either OPN or LPN between January 2016 and May 2020. Seventy patients (45 patients were males and 25 patients were females) with clinical T1N0M0 RCC are included in this study. Patients with missing data $(n=7)$, multiple tumors $(n=1)$, and benign lesions (oncocytoma $n=4$, angiomyolipoma $n=2$, and benign cyst $n=2$ ) were excluded from the study.

The study followed the ethical recommendations of the Declaration of Helsinki and was approved by the Ethics of Research Committee of Prof. Dr. Cemil Taşcıoğlu City Hospital (14.04.2020; number: 119). All the participants in this study have signed an informed consent form.

The mass was diagnosed with computed tomography or magnetic resonance imaging and was treated with OPN or LPN. Fifty patients underwent OPN, and 20 patients underwent LPN.

Renal function was assessed by measuring serum creatinine and estimated glomerular filtration rate (eGFR). The severity of surgical complications was graded according to the modified Clavien classification system. Postoperative histopathology of the tumors, Fuhrman grade, and surgical margin status were recorded. LPN was performed by one experienced urologist, while OPN was done by another two experienced urologists.

Our primary aim was to compare OPN and LPN techniques in terms of the demographic findings, tumor size, PADUA nephrometry score, warm ischemia time (WIT), intraoperative complications, blood loss, operative time, postoperative Clavien-Dindo complications, surgical margins, and hospital stay time. Our secondary aim was to compare the two techniques in terms of AKI (eGFR and creatinine values before the operation and on the postoperative third day).

\section{Statistical Analysis}

Number Cruncher Statistical System (2007) (Kaysville, Utah, USA) was used to perform the statistical analysis with a significance level of $p \leq 0.05$.

We used Student's t-test (years and WIT), Mann-Whitney U test (operation time, PADUA score, hospital stay time, eGFR, creatinine, urea, and hemoglobin ( $\mathrm{Hb}$ ), Pearson's chi-square test (side, male-to-female ratio), Fisher's exact test (bleeding and pathology), Fisher-Freeman-Halton test (Clavien classification and Fuhrman grade), Wilcoxon signed-rank test (creatinine, urea, eGFR, and $\mathrm{Hb}$ ) in our study.

\section{RESULTS}

\section{Demographics}

The study included 70 patients, of which 20 patients (28.6\%) underwent LPN and 50 patients underwent OPN (71.4\%). Fiftyfour patients (77.1\%) were diagnosed with clinical T1a RCC and 16 patients (22.9\%) were diagnosed with clinical T1b RCC. $60 \%(n=42)$ of the patients had right-sided lesions, while $40 \%$ $(n=28)$ had left-sided lesions. The descriptive characteristics of the 70 patients who underwent either OPN or LPN are reported separately in Table 1.

In the OPN group, the operation time and WIT are longer, and the size of the tumor is bigger. Of 70 patients, 19 (27\%) had Clavien-Dindo complications, grades 1-3 [14 patients (28\%) in the OPN group; 5 patients (20\%) in the LPN group; $p=0.451$ ]. Forty-five patients (64.3\%) had a PAUDA score of six to seven, 17 patients (24.3\%) had a score of eight to nine, and eight patients (11.4\%) had a score $\geq 10$.

There is no statistically significant difference between the two techniques in terms of operation time $(p=0.973)$, WIT $(p=0.824)$, size of tumor $(p=0.865)$, PAUDA score $(p=0.195)$, ClavienDindo complications $(p=0.451)$, hospital stay time $(p=0.206)$, preoperative $\mathrm{Hb} \quad(p=0.274)$, and postoperative $\mathrm{Hb}$ values $(p=0.553)$.

The difference between pre- and postoperative $\mathrm{Hb}$ values is statistically significant in OPN and LPN groups ( $p=0.01$ in OPN; $\mathrm{p}=0.043$ in LPN).

Creatinine and eGFR values of patients who underwent either OPN or LPN are shown in Table 2.

Postoperative creatinine increase is statistically different in OPN ( $p=0.001)$ and postoperative eGFR decrease is statistically different in both approaches (OPN: $p=0.001$; LPN: $p=0.001$ ). 
The relationship between PADUA score and WIT, operative time, and Clavien complications is reported in Table 3.

There is a statistically significant difference between PADUA score and operation time in OPN $(r=0.311 ; p=0.028)$. There is no statistically significant difference between PADUA score and operative time, WIT, and Clavien complications in LPN ( $p>0.05)$.

We found surgical margin positivity in two patients who underwent OPN, although we did not see a recurrence during eight and fourteen months of follow-up.
In the OPN group, three patients underwent a transperitoneal $\mathrm{PN}$, and five patients had 12th rib resection. One patient with a PADUA score of 12 had undergone nephrectomy because of bleeding during the operation. Perioperative ultrasonography was performed to locate the tumor in two patients.

In the LPN group, we opted for OPN in one patient with a PADUA score of 11 because of colon injury. The urinary fistula occurred and lasted 18 days in one patient who underwent LPN, and it lasted for 21 days in another patient, who underwent OPN.

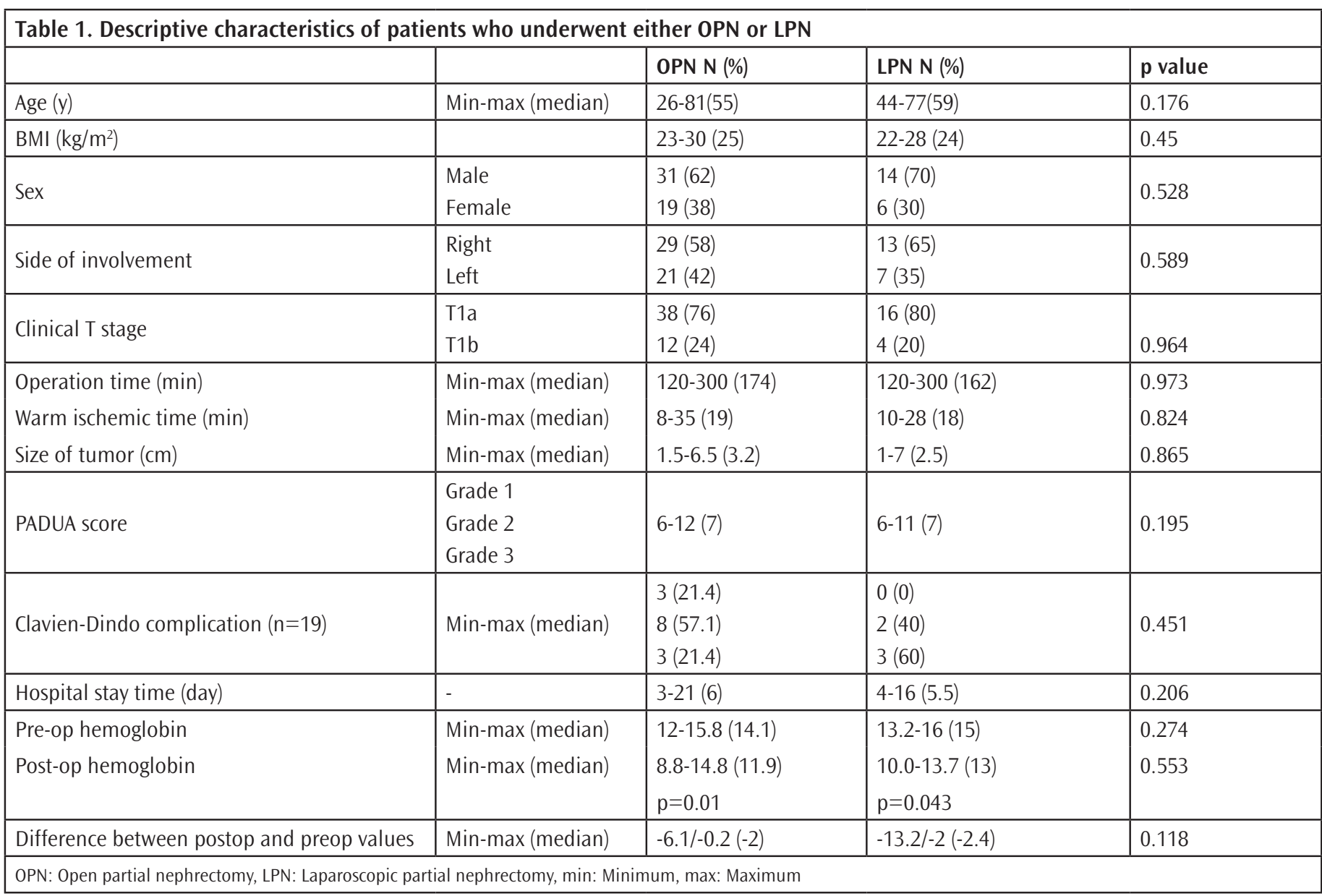

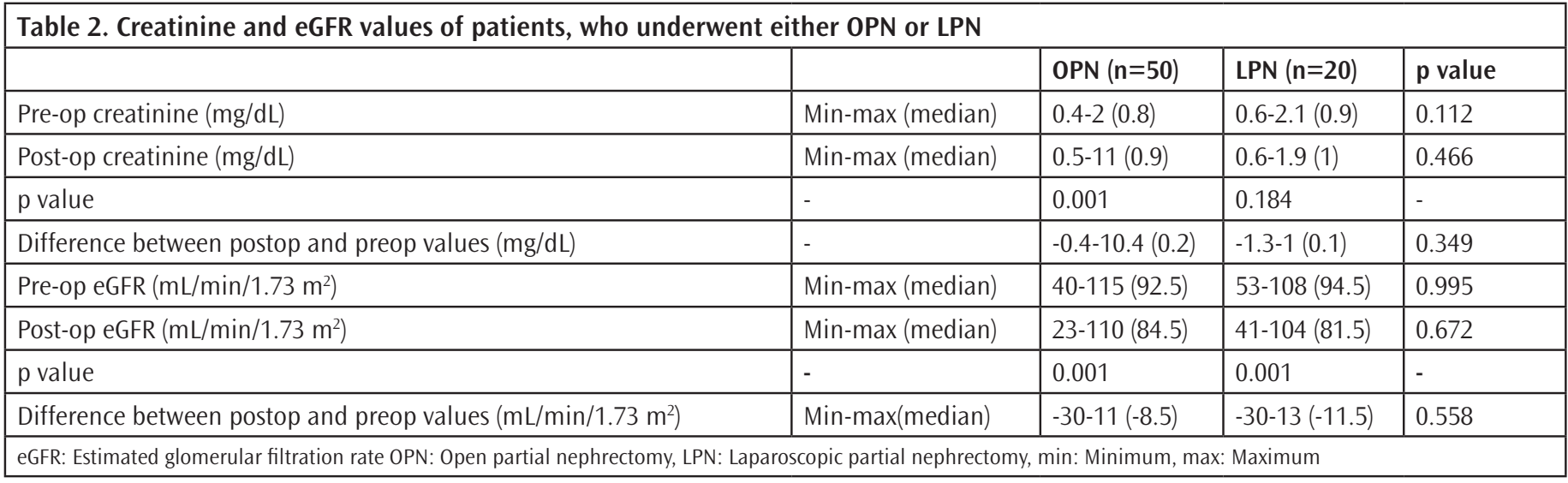


The transfusion rate (intra- and postoperatively) in LPN and OPN group was $10 \%$ and $16 \%$, respectively $(p=0.761)$.

Of 86 patients, eight (9.3\%) had benign lesions (oncocytoma $\mathrm{n}=4$; angiomyolipoma $\mathrm{n}=2$; benign cyst $\mathrm{n}=2$ ).

Of 70 patients, 42 (60\%) had clear cell RCC, 17 (24.4\%) had papillary RCC, and 11 (15.7\%) had chromophobe RCC.

Thirteen (18.6\%) patients had grade 1 Fuhrman, 35 (50\%) patients had grade 2 Fuhrman, and 22 (31.4\%) patients had grade 3 Fuhrman.

\section{DISCUSSION}

Previously, the conventional treatment of kidney tumors was radical nephrectomy. However, PN was performed in patients with small masses with solitary kidney or multiple bilateral tumors.

Today, the success rates, oncological results, and complication rates of $\mathrm{PN}$ are comparable with the results of radical nephrectomy for treating renal tumors. PN can be an open, laparoscopic, or robotic surgery. Due to the need for technical experience and devices to perform a laparoscopic surgery, it took some time for LPN to become an alternative to OPN. We observed that surgical and functional outcomes of LPN and OPN were comparable in our study. Tumor size, operative time, WIT, blood loss, Clavien complications, hospital stay time, eGFR decrease, and creatinine increase were higher in the OPN group, but the difference between the two approaches was not statistically significant in our study cohort.

In the study by Gill et al. (7), in the LPN group, blood loss was less and hospital stay time was shorter, while WIT was longer. In the study by Minervini et al. (11), the median operative time in OPN and LPN is 131.2 min and 143.0 min, respectively, while, in our study, the median operative time in OPN and LPN is $174 \mathrm{~min}$ and $162 \mathrm{~min}$, respectively. Shorter operation time and hospital

\begin{tabular}{|c|c|c|c|c|}
\hline $\begin{array}{l}\text { Table 3. The relations } \\
\text { warm ischemia time, op } \\
\text { complications in OPN and }\end{array}$ & & $\begin{array}{l}\text { en } P \\
\text { me, a }\end{array}$ & $\begin{array}{l}\text { UA sc } \\
\text { d Clav }\end{array}$ & $\begin{array}{l}\text { re and } \\
\text { n-Dindo }\end{array}$ \\
\hline & & Total & $\begin{array}{l}\text { OPN } \\
(n=50)\end{array}$ & $\begin{array}{l}\text { LPN } \\
(n=20)\end{array}$ \\
\hline Clavien-Dindo complications & r & 0.055 & 0.195 & 0.354 \\
\hline$(n=19)$ & $\mathrm{p}$ & 0.824 & 0.504 & 0.559 \\
\hline Wharm icchemic tims (min) & r & -0.035 & 0.067 & -0.287 \\
\hline warm ischemıc tıme (mın) & $\mathrm{p}$ & 0.776 & 0.644 & 0.220 \\
\hline & r & 0.205 & 0.311 & -0.029 \\
\hline Operatıon tıme (mın) & $\mathrm{p}$ & 0.088 & $0.028^{*}$ & 0.905 \\
\hline
\end{tabular}

stay time reduce the perioperative and postoperative morbidity in patients (12). Hospitalization time is shorter in all LPN groups in the literature (13), which agrees with our results.

In our study, Clavien-Dindo complications were higher in the OPN group, which is probably is related to higher PADUA scores and bigger size of tumors.

In Marszalek's study, the overall complication rate in OPN and LPN groups was $22 \%$ and $24 \%$, respectively (13). Overall, the complication rates in the LPN group in the literature range from $9 \%$ to $33 \%$ and in the OPN group range from $4.1 \%$ to $38.6 \%$ (14).

Total complications were not statistically significant between OPN and LPN groups, but intraoperative surgical complications were higher for the OPN group in A. Minervini's study $(11,13)$.

WIT (minimum-maximum-median) in the OPN and LPN groups was 8-35 (19) min and 10-28 (18) min, respectively $(p=0.824)$ in our study group. The LPN groups had a significantly longer WIT in many studies in the literature (11), although some studies reported shorter WIT in the LPN groups $(7,13)$. Bravi et al. (15) showed that LPN and robotic PN had a longer ischemia time than OPN. In our study, shorter WIT in the LPN group may be related to the surgeon's experience and complexity of the tumor.

The surgical margin was positive in two patients who underwent OPN in our study. A higher PADUA score and a larger size of tumor can affect this finding. A multicenter analysis of LPN showed positive surgical margins (PSM) in $1.8 \%-2.4 \%$ of the patients (16). In Kwon's et al. (17) study, the PSM rate of OPN is 7\%. In Andrea Minervini's study, the incidence of PSM was not significantly different between both groups (3.5\% for OPN and $3 \%$ for LPN) (11). Bravi et al. (15) showed that minimally invasive approaches did not affect the risk of PSM when compared with open surgery. Only $4 \%$ of patients with PSM will develop a local recurrence (17). Our two patients with PSM did not experience recurrence during the eight and fourteen months of followup. However, we must follow up these patients closely.

In our study, the difference between the two approaches in terms of pre- and postoperative $\mathrm{Hb}$ values is statistically significant ( $p=0.001$ in OPN and $p=0.043$ in LPN). The decrease in $\mathrm{Hb}$ values postoperatively in both approaches is not statistically different $(p=0.118)$. These findings correlate with the literature.

In Marszalek et al.'s (13) study, the transfusion rate was $6 \%$ in the LPN group and $11.0 \%$ in the OPN group $(p=0.2)$. The transfusion rate in our LPN group was 10\% and in the OPN group 16\% ( $p=0.761)$. In Minervini et al.'s (11) study, the mean intraoperative blood loss was slightly higher for the OPN group, 
but it was not statistically significant (221 cc vs. 164 cc). In White et al.'s (18) study, the median blood loss was $200 \mathrm{~mL}(100 \mathrm{~mL}-375$ $\mathrm{mL}$ ) in patients who underwent robotic PN.

The increase in postoperative creatinine $(p=0.001 ; p<0.01)$ and decrease in eGRF $(p=0.001 ; p<0.01)$ are statistically different in our OPN group. Preoperative and postoperative creatinine and eGFR values are not statistically different in OPN and LPN groups (creatinine: $p=0.349$; eGRF: $p=0.558 ; p>0.05$ ).

AKI develops in approximately $20 \%$ of patients after PN (4). AKI can be temporary and may take up to $24-72 \mathrm{~h}$ or can be persistent (19). Bravi et al. (15) demonstrated that AKI is associated with long-term renal function and duration of the injury. The longer the duration of AKI (specifically exceeding the third day), the more reduced the long-term renal function (15).

Jimenez-Romero et al. (20) showed that, in the LPN group, patients weighing more than $84 \mathrm{~kg}$, the tumor size being larger than $4 \mathrm{~cm}$, WIT exceeding $26 \mathrm{~min}$, operation time exceeding 200 min are more likely to cause renal function impairment after the operation. In their study, 18 patients (37.5\%) preserved their renal function and 30 (62.5\%) had a renal function impairment postoperatively (20).

Martín et al. (21) stated that if WIT exceeds 25 min, each additional minute increases renal function deterioration (RFD) by $5 \%-6 \%$. The protected renal parenchyma is an important factor in RFD (21). The median size of the tumors in patients with deteriorated renal function was $1.1 \mathrm{~cm}$ larger than that in patients without RFD (20).

Marszalek et al. (13) found that the decrease in eGFR rate in both groups was similar $(p=0.8)$. Minimally invasive techniques (both laparoscopic and robotic) had a lower risk of AKI than open surgery (both $p<0.0001)$ (15). Our findings in the LPN group are correlated with this study.

White et al. (18) found median eGFR decrease of $11.1 \mathrm{~mL} /$ $\mathrm{mm} / 1.73 \mathrm{M} 2$ in his robotic PN group, whose patients had renal masses with a nephrometry score of $\geq 7$. In our study, the median GFR decrease is 8.5 and 11.5 in OPN and LPN groups, respectively. The mechanisms determining AKI after $\mathrm{PN}$ is not fully understood (22).

The age of the patient, preoperative kidney function, renal perfusion during surgery, WIT, operative time, operation technique, intraoperative blood loss, resected tumor volume, type and-duration of anesthesia, and surgeon's experience may affect AKI. If $A K I$ is not transient ( $\geq 3$ day), the kidney's function can be worse in the long-term.
There is a statistically significant difference between PADUA score and operative time in the OPN group $(r=0.311 ; p=0.028)$ in our study cohort. The other relationships in both approaches are comparable.

The possibility of having a positive trifecta in patients who have a PADUA score $<10$ and treated robotically was higher than in patients who underwent OPN and LPN (15).

Altunrende et al. (23) found a correlation between total RENAL nephrometry score and WIT after robotic PN (Spearman's correlation coefficient: $0.54 ; p<0.0001)$. In this study, they declared that posteriorly located tumors require complete mobilization of the kidney, and this increases the adjacent tissue damage (23).

When PADUA score increases, the complexity of the tumor and complications, operation time, hospital stay time, and bleeding increase. PN in patients with high PADUA score $(\geq 10)$ is usually performed by open surgery (15). The rate of converting from PN to radical nephrectomy during the operation was approximately $5 \%$ regardless of the surgical technique (24). The rate of converting to open surgery in LPN and robotic PN was 7 (1\%) and $1(<1 \%)$, respectively, in this study (15).

We changed the decision from $\mathrm{PN}$ to radical nephrectomy in one patient in the OPN group because of bleeding and a PADUA score of 12. We converted LPN to OPN in one patient because of bleeding and injury of the colon with a PADUA score of 11.

The urinary fistula occurred and lasted 18 days in one patient, who underwent LPN, and lasted 21 days in another patient, who underwent OPN.

\section{CONCLUSION}

LPN is an alternative technique to OPN for treatment of clinical T1 RCC by experienced urologists in experienced clinics. Postoperative creatinine increase in OPN is more obvious. All perioperative outcomes in OPN and LPN are comparable to each other in T1 RCC.

\section{Ethics}

Ethics Committee Approval: The study followed the ethical recommendations of the Declaration of Helsinki and was approved by the Ethics of Research Committee of Prof. Dr. Cemil Tașcıoğlu City Hospital (14.04.2020; number: 119).

Informed Consent: All the participants in this study have signed an informed consent form.

Peer-review: Externally and internally peer-reviewed. 


\section{Authorship Contributions}

Surgical and Medical Practices: E.M., Concept: E.M., Design: A.A., Data Collection or Processing: E.M., A.A., Analysis or Interpretation: E.M., A.A., Literature Search: E.M., Writing: E.M.

\section{REFERENCES}

1. European Network of cancer Registries: Eurocimversion 4.0.2001; Lyon, France.

2. Doeuk N, Guo DY, Haddad R, Lau H, Woo HH, Bariol S, et al. Renal cell carcinoma: stage, grade and histology migration over the last 15 years in a large Australian surgical series. BJU Int 2011;107:1381-5.

3. Capitanio U, Montorsi F. Renal cancer. Lancet 2016;387:894-906.

4. Martini A, Cumarasamy S, Beksac AT, Abaza R, Eun DD, Bhandari A, et al. A Nomogram to Predict Significant Estimated Glomerular Filtration Rate Reduction After Robotic Partial Nephrectomy. Eur Urol 2018;74:833-9.

5. Becker F, Siemer S, Hack M, Humke U, Ziegler M, Stöckle M. Excellent long-term cancer control with elective nephron-sparing surgery for selected renal cell carcinomas measuring more than $4 \mathrm{~cm}$. Eur Urol 2006;49:1058-63; discussion 1063-4.

6. Uzzo RG, Novick AC. Nephron sparing surgery for renal tumors: indications, techniques and outcomes. J Urol 2001;166:6-18.

7. Gill IS, Kavoussi LR, Lane BR, Blute ML, Babineau D, Colombo JR Jr, et al. Comparison of 1,800 laparoscopic and open partial nephrectomies for single renal tumors. J Urol 2007;178:41-6.

8. Chang KD, Abdel Raheem A, Kim KH, Oh CK, Park SY, Kim YS, et al. Functional and oncological outcomes of open, laparoscopic and robotassisted partial nephrectomy: a multicentre comparative matched-pair analyses with a median of 5 years' follow-up. BJU Int 2018;122:618-26.

9. Pereira J, Renzulli J 2nd, Pareek G, Moreira D, Guo R, Zhang Z, et al. Perioperative Morbidity of Open Versus Minimally Invasive Partial Nephrectomy: A Contemporary Analysis of the National Surgical Quality Improvement Program. J Endourol 2018;32:116-23.

10. Ljungberg B, Albiges L, Bensalah K, Bex A, Giles RH, Hora M, et al. EAU guidelines on renal cell carcinoma 2018. In: European Association of urology Guidelines. 2019 Edition. Volume presented at the EAU Annual Congress Copenhagen 2018. October 15,2019. https:// uroweb.org/wp-content/uploads/EAU-RCC-Guidelines-2018-large-text. pdf TheNetherlands: European Association of Urology Guidelines Office;2018

11. Minervini A, Siena G, Antonelli A, Bianchi G, Bocciardi AM, Cosciani Cunico $S$, et al. Open versus laparoscopic partial nephrectomy for clinical T1a renal masses: a matched-pair comparison of 280 patients with TRIFECTA outcomes (RECORd Project). World J Urol 2014;32:257-63.
12. Routh JC, Bacon DR, Leibovich BC, Zincke H, Blute ML, Frank I. How long is too long? The effect of the duration of anaesthesia on the incidence of non-urological complications after surgery. BJU Int 2008;102:301-4.

13. Marszalek M, Meixl H, Polajnar M, Rauchenwald M, Jeschke $K$ Madersbacher S. Laparoscopic and open partial nephrectomy: a matched-pair comparison of 200 patients. Eur Urol 2009;55:1171-8.

14. Porpiglia F, Volpe A, Billia M, Scarpa RM. Laparoscopic versus open partial nephrectomy: analysis of the current literature. Eur Urol 2008;53:732-42; discussion 742-3.

15. Bravi CA, Larcher A, Capitanio U, Mari A, Antonelli A, Artibani W, et al. Perioperative Outcomes of Open, Laparoscopic, and Robotic Partial Nephrectomy: A Prospective Multicenter Observational Study (The RECORd 2 Project). Eur Urol Focus 2021;7:390-6.

16. Canes D. Long-term oncological outcomes of laparoscopic partial nephrectomy. Curr Opin Urol 2008;18:145-9.

17. Kwon EO, Carver BS, Snyder ME, Russo P. Impact of positive surgical margins in patients undergoing partial nephrectomy for renal cortical tumours. BJU Int 2007;99:286-9.

18. White MA, Haber GP, Autorino R, Khanna R, Hernandez AV, Forest $S$, et al. Outcomes of robotic partial nephrectomy for renal masses with nephrometry score of $\geq 7$. Urology 2011;77:809-13.

19. Chawla LS, Bellomo R, Bihorac A, Goldstein SL, Siew ED, Bagshaw SM, et al. Acute kidney disease and renal recovery: consensus report of the Acute Disease Quality Initiative (ADQI) 16 Workgroup. Nat Rev Nephrol 2017:13:241-57.

20. Jimenez-Romero ME, Moreno-Cortes JC, Canelon-Castillo EY, DiezFarto S, Santotoribio JD. Predictive Factors of Renal Function in Partial Laparoscopic Nephrectomy in Patients with a Kidney Tumor. Curr Urol 2019;13:150-6.

21. Martín OD, Bravo H, Arias M, Dallos D, Quiroz Y, Medina LG, et al. Determinant factors for chronic kidney disease after partial nephrectomy. Oncoscience 2018;5:13-20.

22. Bravi CA, Vertosick E, Benfante N, Tin A, Sjoberg D, Hakimi AA, et al. Impact of Acute Kidney Injury and Its Duration on Long-term Renal Function After Partial Nephrectomy. Eur Urol 2019;76:398-403.

23. Altunrende F, Laydner H, Hernandez AV, Autorino R, Khanna R, White MA, et al. Correlation of the RENAL nephrometry score with warm ischemia time after robotic partial nephrectomy. World J Urol 2013;31:1165-9.

24. Petros FG, Keskin SK, Yu KJ, Li R, Metcalfe MJ, Fellman BM, et al. Intraoperative Conversion From Partial to Radical Nephrectomy: Incidence, Predictive Factors, and Outcomes. Urology 2018;116:114-9. 